\title{
Investigación Acción En Un Entorno Educativo: Programa Para Promover Creencias Positivas Hacia Una Cultura De Paz Y De Prevención A La Violencia.
}

\author{
Dra. Analinnette Lebrija Trejos \\ Universidad Especializada de las Américas, Panamá
}

doi: 10.19044/esj.2016.v12n19p127 URL:http://dx.doi.org/10.19044/esj.2016.v12n19p127

\begin{abstract}
The objective of the research is validating a program to promote positive beliefs towards a culture of peace and prevention of school violence from a psychological perspective and health promotion. Beliefs are understandings and assumptions about the world that are perceived as truths and that underlie the attitudes and decision making of individuals. We carried out a total of 6 workshops, of two hours each given weekly, and two evaluation sessions, to 43 students divided into a study group and a control group, with a simple random sample type. During the week, we used process-measuring instruments. The workshops were based on competence of life and education for peace. The study was conducted in a school located in Panama City. The subjects were teenagers from eighth grade (mid-school), aged 13 to 15 years. The study is a pretest - posttest study with control group, action research that allowed reflection and participation of students on the issue of violence. Quantitative and qualitative results indicate that the program managed to positive change the beliefs of students towards violence; the results and the theoretical background support that a change of attitudes in the long-term requires that these types of Programs are embedded in the academic process as a constant and systematic element.
\end{abstract}

Keywords: Prevention program of school violence, psychological paradigm, health promotion, beliefs, skills, high school students

\section{Resumen}

Investigación cuyo objetivo es validar un programa para promover creencias positivas hacia una cultura de paz y prevención de la violencia escolar desde una perspectiva psicológica y de promoción a la salud; se fomenta la modificación de las creencias, que son entendimientos y premisas acerca del mundo, percibidas como verdades que fundamentan la toma de decisiones y actitudes del individuo. Se realizan 6 talleres, de dos 
horas cara uno, impartidos uno por semana, así como dos sesiones de evaluación, en 43 estudiantes divididos en grupo control y grupo estudio, con un tipo de muestra aleatoria simple. Se utilizan instrumentos de medición de proceso durante la semana. Los talleres están fundamentados en competencias para la vida y educación para la paz. El estudio se realizó en un colegio Ciudad de Panamá. Los sujetos participantes fueron adolescentes de octavo de pre-media, con edades entre 13 y 15 años. Es un estudio pretest - postest, con grupo control, investigación acción que permitió la reflexión y participación de los estudiantes en la problemática de la violencia. Los resultados cuantitativos y cualitativos, indican que el Programa logró modificar positivamente las creencias de los estudiantes hacia la violencia; los resultados y los antecedentes teóricos sustentan que para modificar actitudes a largo plazo se requiere que este tipo de programas estén insertos en el proceso académico como un elemento constante y sistemático.

Palabras claves: Programa de prevención de la violencia escolar, paradigma psicológico, promoción a la salud, creencias, competencias, adolescentes de octavo de secundaria

\section{Introducción}

Las condiciones y requisitos para la salud son la paz, la educación, la vivienda, la alimentación, un ecosistema estable, la justicia social y la equidad; estas circunstancias son una perspectiva de la promoción a la salud, que se describe en la conferencia de la Organización Mundial de la Salud, realizada en Otawa - Canadá, que contempla la cultura de paz y por tanto la prevención a la violencia como un aspecto fundamental. (OMS, 2002, 1996, 1997, 1998).

Muchos adolescentes aprenden a solucionar los problemas de forma violenta dentro de un marco de normalidad y juego, con actitudes agresivas como golpes, palabras soeces, empujones, burlas, bromas pesadas, etc. (Eljach, 2011). Este es un problema mundial declarado en el seno del Organismo de Naciones Unidas, donde se han propuesto investigaciones importantes como la que se manifiesta en el documento, acabar con la violencia contra los niños, niñas y adolescentes (Pinhjeiro, 2007).

La Organización Mundial de la Salud propone que en el siglo XXI, la promoción de la salud debe formar parte de una estrategia educativa fundamental para alcanzarla; promover un estado de completo bienestar físico, mental y social, y no solamente la ausencia de enfermedad o dolencia.

La salud es un recurso de la vida diaria, no el objetivo de la vida. Se trata de un concepto positivo que acentúa los recursos sociales y personales, así como las aptitudes físicas (OMS, 1996). 
Según UNICEF (2011), la violencia escolar en América Latina y el Caribe es un problema de enorme gravedad; sus estudios revelaron que en una población aproximada de 800 mil estudiantes, entre 400 mil y 550 mil de ellos, esto es, entre el 50\% y 69\% estudiantes, en algún momento en las aulas de clase, pudieron ser víctimas de violencia.

Panamá no escapa a esa realidad; el nivel de violencia escolar, según estudio realizado por el Centro de Estudio de Acción Social Panameña (CEASPA, 2013), aumenta cada día; no es extraño escuchar y leer en los medios de comunicación panameño casos de violencia entre adolescentes, dentro y fuera del plantel educativo.

Como una forma de coadyuvar en la problemática manifiesta, se propuso y desarrolló un programa formativo de prevención de la violencia y promoción de la cultura de paz, desde la perspectiva psicológica de modificación de las creencias y de fomento a la salud, para adolecentes de $8^{\circ}$ nivel en Panamá. La promoción de la cultura de paz se consideró como un recurso que permita a las personas llevar una vida individual y social para lograr un fin saludable, y no como una concepción abstracta.

Como el estudiante no se conoce así mismo, se trabajaron aspectos como autoestima, auto concepto, comunicación asertiva, relaciones interpersonales, emociones, sentimientos, pensamiento crítico, entre otros aspectos, que conforman el desarrollo psicológico de un individuo y que son vitales para un crecimiento sano (Tuvilla, 2004). Se buscaron soluciones en el proceso de desarrollo de los estudiantes para atender la problemática en el escenario en el que ocurre la violencia diariamente. Se partió del hecho de que las relaciones interpersonales de los estudiantes, así como la comunicación entre ellos, se fusionan con costumbres violentas que son percibidas como adecuadas.

El problema es estructural y debe ser abordado desde un punto de vista interdisciplinario en el que se incluyan la política, la educación, la salud, el derecho, la psicología, los medios de comunicación, el trabajo social y la seguridad ciudadana tanto en el análisis, como en el tratamiento. Se planteó que las medidas para la solución del problema deben situarse en tres ámbitos: el de la prevención, el de consecuencias a las conductas violentas y el asistencial. Se les expuso a los docentes y a sus padres.

\section{Marco teórico}

El programa desarrollado en la investigación, para estudiantes de $8^{\circ}$ nivel en Panamá, se centró en el fomento de una cultura de paz con modificación en las creencias relativas a la violencia escolar desde una perspectiva psicológica de promoción de la salud de los adolecentes; violencia escolar entre pares en una de sus principales modalidades, el bullying, (agresiones físicas, insultos, burlas, amenazas, intimidaciones, 
aislamiento sistemático, vandalismo) (Gómez, et al., 2007, Kerman, 2015, 2016).

Para la erradicación de la violencia se consideraron autores como OMS (1996,1997,1998), INJUVE (2010), Ortega (2000,2003), Bandura (1996), Del Rey y Ortega (2001), Morollon (2001), Bisquerra (2003), DíazAguado (2003, 2004), quienes manejan componentes cognitivos, psicológicos que pueden ser modificados mediante procesos educativos, afectivos o evaluativo. El riesgo de la violencia aumenta cuando se asocia a valores o a personas con las que el sujeto se identifica y conductual cuando se producirse por la falta de habilidades que permitan resolver los conflictos sociales. Todo lo anterior involucrando las competencias para la vida y el bienestar que son la capacidad para adoptar comportamientos apropiados y responsables.

La violencia escolar tiende a originar problemas que se repiten y prolongan durante cierto tiempo y supone un abuso de poder de un alumno contra una víctima que se encuentra indefensa que no sabe salir de esta situación. La violencia entre iguales se mantiene debido a la ignorancia o pasividad de las personas que rodean a los agresores y a las víctimas, sin intervenir directamente. (Díaz -Aguado, 2004).

Para la prevención de la violencia, el programa tomó recomendaciones de UNICEF (2011) para lograr entornos escolares seguros y propicios para el aprendizaje, la Cruz Roja (2003) que describe estrategias educativas para la prevención a la violencia, promoviendo la comunicación, el diálogo, la medicación de conflictos, y habilidades sociales. Para la tolerancia, y la mediación, fueron considerados, Torrego (2000), el Programa "Convivir es vivir", en el Comunicado de Madrid en 1999, Ortega, (2003, 2004) con el Programa la convivencia escolar, qué es y cómo abordarla y El Educar la convivencia para prevenir la violencia.

Las creencias, que inciden en el fenómeno de la violencia de los adolescentes, se estudiaron en Green, (1971), Hart (1989), Pajares (1992), Ortega y Gasset, (1989), Young (1969), Corozzo, (2010) y Kerman (2015).

Los ejes transversales intervenidos son educación para la paz, educación para la vida en sociedad y para la convivencia con modificación en las creencias; se crearon talleres para trabajar los conceptos, desde una perspectiva psicológica de promoción de la salud y la prevención de la violencia en adolescentes de $8^{\circ}$ de media académica, se estructuró y desarrolló el programa para el fomento de una cultura de paz.

\section{Planteamiento del problema de investigación.}

Se hace evidente la necesidad de abordar la prevención de conflictos en el ambiente escolar, con el componente familiar presente en el problema. 
En la prevención intervienen tres agentes educativos importantes: la familia, la escuela y los medios de comunicación. En esta investigación no se involucraron a los medios de comunicación.

La familia que debe ofrecer un espacio de encuentro gratificante y potenciador de las capacidades y posibilidades de todas las personas que lo integran.

La escuela que debe ser un espacio ideal para fomentar valores tales como el respeto, la igualdad dentro de la diversidad, la comprensión hacia las diferencias personales, la adquisición de un espíritu crítico (Tuvilla, 2004).

\section{Problema de Investigación.}

El problema de investigación se formula explícitamente del modo siguiente: si se pretende favorecer un cambio de la violencia en el aula a partir de la modificación de las creencias en ese contexto ¿cuáles son las potencialidades y las limitaciones de un Programa de prevención de la violencia escolar con el fomento de una cultura para la paz? Para abordar este problema se desarrolló y se evaluó un programa basado en 6 talleres de intervención.

\section{Metodología}

\section{Objetivos}

\section{Objetivo General}

Validar un programa para promover creencias positivas hacia una cultura de paz y prevención de la violencia escolar desde una perspectiva psicológica y de promoción a la salud.

\section{Objetivos Específicos}

- $\quad$ Diseñar y validar herramientas para la evaluación diagnóstica y acompañamiento del estudiante durante el programa.

- $\quad$ Analizar el nivel de violencia entre iguales.

- Describir las situaciones sufridas por los adolescentes como victimas, como agresor y como espectador.

- Analizar las creencias de los adolescentes con respecto a las conductas violentas, relaciones interpersonales, comunicación, valores, manejo de sentimientos y emociones.

- $\quad$ Evaluar objetivamente la efectividad del programa aplicado.

\section{Hipótesis}

\section{Hipótesis I :}

Ho: El programa de prevención a la violencia y promoción de la cultura de paz, disminuye la violencia entre iguales.

Hi: El programa de prevención a la violencia y promoción de la cultura de paz, no mejora la violencia entre iguales. 


\section{Hipótesis II :}

Ho: El programa de prevención a la violencia y promoción de la cultura de paz, mejora las situaciones sufridas como victimas, agresores o espectadores.

Hi: El programa de prevención a la violencia y promoción de la cultura de paz, no mejora las situaciones sufridas como victimas, agresores o espectadores.

\section{Hipótesis III :}

Ho: El programa de prevención a la violencia y promoción de la cultura de paz, promueve creencias en contra de conductas violentas.

Hi: El programa de prevención a la violencia y promoción de la cultura de paz, no promueve creencias en contra de conductas violentas.

\section{Diseño de investigación y tipo de estudio.}

Es un diseño pretest - postest con grupo control, el cual no se tiene el control de todas las variables, al estar trabajando en un contexto educativo real, expuesto a muchas eventualidades; nos permite un enfoque más práctico que teórico y la auto reflexión a través de la técnica de portafolio como instrumento cualitativo de evaluación de proceso (Guerrero, 2015).

La parte cualitativa del estudio se desarrolla a través de una investigación acción que liga el enfoque experimental del estudio con la acción social-educativa de modificación de creencias hacia la violencia. Se promueve una reflexión de las actitudes de los estudiantes y las experiencias vividas, enfocadas a la modificación de las creencias hacia el concepto de violencia y sus implicaciones en las relaciones interpersonales del estudiante dentro del colegio (Murillo, 2011).

El tipo de estudio es descriptivo, cualitativo y cuantitativo, permitiendo de esta forma analizar resultados con base en un nivel de confiabilidad estadística, como describir aquellas características del estudio útiles y medulares que no se puedan obtener con los instrumentos del pretest - postest. (Hernández, 2010).

\section{Población. Sujetos, tipo de muestra, criterios de inclusión de la muestra}

Sujetos: Son los estudiantes de un colegio panameño de $8^{\circ}$ grado de premedia en un rango de edad de 14 a 15 años. El colegio cuenta con tres grupos de la característica deseada por lo que se eligieron dos grupos con un muestreo aleatorio simple, el grupo control y el grupo de estudio. La participación de los alumnos fue voluntaria previa autorización de los padres. Se excluyeron alumnos con más de 10 \% de inasistencia, y es la única característica de selectividad, todos presentan problemas de violencia escolar, de acuerdo con lo descrito psicológico del colegio. Se trabajo con un total de 43 estudiantes distribuidos en dos grupos, grupo control 10 mujeres 
y 12 hombres, y grupo estudio 12 mujeres y 9 hombres.

\section{Variables.}

\section{Variable Independiente: Programa de Prevención a la violencia}

Definición conceptual: es un programa de intervención que consta de 8 sesiones de dos horas cada una por semana, la primera y la última son de evaluación y las 6 restantes son sesiones-taller de dos horas semanales, basados en competencias para la vida, que fomentan la modificación de creencias y estrategias de solución de problemas necesaria para una cultura de paz y prevención de la violencia escolar.

Definición operacional: se medirá a través de la modificación de la violencia escolar y las creencias hacia la violencia.

\section{Variable dependiente 1: Violencia escolar (Bullying)}

Definición Conceptual: violencia escolar es la acción u omisión intencionadamente dañina ejercida entre miembros de la comunidad educativa (alumnos, profesores, padres, personal subalterno) y que se produce dentro de los espacios físicos que le son propios a las instalaciones escolares, o bien en otros espacios directamente relacionados con lo escolar (alrededores de la escuela o lugares donde se desarrollan actividades extraescolares.

Definición Operacional: la violencia escolar se medirá a través de los siguientes criterios: Calidad de contextos y relaciones; Situaciones sufridas como víctimas en la escuela; Situaciones vividas como agresor en la escuela; Situaciones vividas como espectador en la escuela; violencia entre igual.

\section{Variable dependiente 2: Creencias hacia la violencia}

Definición Conceptual: Son verdades personales indiscutibles sustentadas por cada uno, derivadas de la experiencia o de la fantasía que tienen un fuerte componente evaluativo y afectivo. Se manifiestan a través de declaraciones verbales o de acciones que las justifican, en este caso con respecto a la violencia.

Definición Operacional: Se midieron con base en: conductas violentas; relaciones interpersonales; comunicaciones, valores; manejo de sentimientos y emociones.

\section{Instrumentos y herramientas de recolección de datos}

Se utilizaron instrumentos para antes y después de la aplicación del Programa, así como instrumentos de proceso que nos permiten medirlo. El cuestionario de actitudes hacia la diversidad y la violencia (CADV), que evalua la justificación de la violencia entre iguales como reacción y valentía 
y un cuestionario de evaluación de la violencia sufrida como víctimas, como agresor y como espectador.(CEVEO). Ambos propuestos por DíazAguado(2004); Cuestionario cerrado de medición de creencias sobre, conductas violentas, comunicación, valores, manejo de sentimientos y emociones, relaciones interpersonales y solución de problemas. Propuesto por Lebrija (2014); Cuestionarios de autoevaluación; El portafolio como instrumento de evaluación de proceso (metas, experiencia personal, dudas, soluciones, cuestionarios de opinión y tareas).

\section{Procedimiento}

El programa se desarrolla en tres etapas: Etapa uno: Evaluación inicial. Detección de necesidades. Etapa dos: Talleres y evaluación de proceso. Etapa tres: Evaluación final.

Los datos se analizaron utilizando estadística descriptiva e inferencial, desde un enfoque mixto cualitativo cuantitativo a través de la utilización de pruebas paramétricas de comparación de medias, se utiliza una prueba $\mathrm{T}$ para muestras relacionadas porque permite analizar los cambios antes y después de la intervención; dichas medias permitieron analizar la eficiencia del programa de intervención e instrumentos de evaluación de proceso, portafolio,(metas, experiencia personal, cuestionarios de opinión, tareas); los cuales permitieron el análisis cualitativo de los datos, describiendo el cambio de creencias, así como el aprendizaje y las opiniones de los adolescentes participantes. Finalmente, se evaluaron los logros y se dieron los resultados a los alumnos, profesores y autoridades del colegio.

\section{Componentes académicos del programa}

Son 6 talleres de 1 hora 40 minutos (2 horas de clase), de tipo vivencial y presencial.

Los temas de los talleres fueron: 1. Cultura de paz y diversidad; 2. Comunicación asertiva. 3. Relaciones interpersonales sanas. 4. Manejo de sentimientos y emociones. 5. Toma de decisiones y solución de problemas. 6. Pensamiento crítico y respeto a la diversidad.

Cada taller se estructuró en secciones: Presentación, (activación del conocimiento); Establecimiento de Metas (evaluación de logros). Análisis del tema y actividades didácticas, (nuevo conocimiento). Evaluación (análisis de logros).

En cada taller se dejaron tareas para ir midiendo la efectividad por ejemplo:

Tarea del taller 2: Describe cómo fue tu relación con tus padres, amigos, hermanos, esta semana.

Tarea del taller 3: Qué mensajes dijiste en la semana de forma inadecuada y cómo los corregiste. Leer la lectura de las Asamblea de 
Herramientas y hacer un escrito de una cuartilla máximo expresando qué entendiste.

Tarea del taller 4: Analiza y describe los sentimientos y emociones que experimentaste durante la semana. Puedes escribir, dibujar o representarlo de alguna forma.

Tarea del taller 5: Analiza y describe qué problemas tuviste en la semana y cómo los resolviste.

\section{Resultados y discusión}

Los resultados cuantitativos y cualitativos, indican que el Programa logró modificar las creencias de los estudiantes hacia la violencia; Después del Programa los alumnos tienen creencias que justifican menos la violencia

Análisis Pretest - Postest del programa de intervención a través de la comparación de medias del grupo estudio y el grupo control.

$>\quad$ Violencia entre iguales (CADV).

$>$ Situaciones sufridas como víctimas, como agresor y como espectador. (CEVEO).

$>\quad$ Creencias de los adolescentes hacia las conductas violentas relaciones interpersonales, comunicación, valores, manejo de sentimientos y emociones.

El resultado de la pruebas $\mathrm{T}$ con respecto a la violencia escolar entre iguales muestran diferencias significativas entre las medias antes y después del programa de prevención a la violencia, pues se obtuvo un nivel de significancia $(\rho>0.00)$ significativo. Las pruebas estadísticas con respecto a la violencia entre iguales del grupo control no muestran diferencias significativas entre las media antes y después del programa, pues se obtuvo un nivel de significancia $(\rho>0.213)$ no significativo. Lo que indica que el programa con respecto a la violencia entre iguales logro modificar las creencias de los estudiantes con el conjunto de tareas y análisis realizados en los talleres.

Por otro lado, si se analizan las medias de las creencias, comparándolas con el criterio: “a menor creencias más adecuadas para prevenir la violencia”, se puede argumentar que los estudiantes mostraron creencias positiva haca la prevención de la violencia; la media del grupo estudio se modificó positivamente de 4,3 (ds: .79) a 2,1 (ds: .71) después el programa.

Con respecto a la violencia escolar en situaciones sufridas como víctimas, como agresor y como espectador. Las prueba $\mathrm{T}$ no muestra diferencias significativas entre las media antes y después del programa del grupo control, pues se obtuvo un nivel de significancia de $(\boldsymbol{\rho}>0,323)$, no significativo. 
En cambio el grupo estudio si muestra diferencias significativas entre las media antes y después del programa, pues se obtuvo un nivel de significancia de $(\rho>0.00)$ significativo.

Si se analizan las medias, comparándolas con el criterio: a menor, creencias más adecuadas para prevenir la violencia, se puede argumentar que los estudiantes mostraron creencias más adecuadas para la prevención de la violencia; la media se modificó positivamente de 3,4 con una (ds: 0,14 ) a 2,5. (ds: .27) Lo que indica que los talleres lograron modificar las creencias de los estudiantes con respecto a la cantidad de situaciones vividas como victima, agresor o espectador de la violencia.

Los resultados estadísticos con respecto a las creencias de los adolescentes hacia las conductas violentas, relaciones interpersonales, comunicación valores, manejo de sentimientos y emociones, muestran que en el grupo control las creencias fueron más negativas en la segunda medición que en la primera medición, es decir dos meses antes. Pero este cambio no fue significativo, $(\boldsymbol{\rho}>\mathbf{0 . 4 2 6})$.

En el grupo estudio las pruebas estadísticas con respecto a las creencias, muestran diferencias significativas entre las media antes y después del programa, pues se obtuvo un nivel de significancia de $(\rho>$ 0.00).

Si se analizan las medias, comparándolas con el criterio: a mayor puntuación de creencias más adecuadas para prevenir la violencia”, podemos argumentar que los estudiantes mostraron creencias más adecuadas para la prevención de la violencia; la media se modificó positivamente de 1.8 (ds:.16) a 2.2 (ds:.21).

Análisis estadístico por pregunta de los datos obtenidos antes y después del Programa

Análisis. Violencia entre Iguales

Cuando un amigo/a agrede a alguien que le ha molestado debes ponerte de parte de tu amigo/a?

Después del Programa la forma de pensar de los estudiantes se modificó, antes del programa el 23,8\% pensaban que si un amigo agrede a alguien no debes ponerte de su parte, después del programa aumentó al $28,6 \%$, lo que nos indican que comprendieron que si un amigo esta agrediendo, no debes hacer lo mismo. Los estudiantes modificaron sus creencias positivamente.

\section{Si un amigo (a) tiene miedo crees que es un cobarde?}

Las creencias se modificaron positivamente; más estudiantes después del Programa pensaron que si un compañero tiene miedo no quiere decir que es un cobarde. La respuesta del mínimo acuerdo se modificó de 4,8\% antes 
del programa al 3,81\% después del programa. El 42,9 \% tienen algunas dudas con respecto a si alguien tiene miedo quiere decir que es un cobarde, lo que refleja que se tiene que seguir trabajando en este punto.

\section{Crees que la violencia forma parte de la naturaleza humana?}

Los estudiantes después del Programa tuvieron creencias más adecuadas con respecto a que la violencia no forma parte de la naturaleza humana. La respuesta al cuestionamiento se modificó de 4,8\% antes del programa a 3,33\% después del Programa.

\section{Es correcto pegar a alguien que te ha ofendido?}

Después del Programa los alumnos justificaron menos la creencia de que si alguien te ofende es correcto pegarle. Después del Programa el 33,3\% optó por la opción “estas de acuerdo” y el 42,9\% por la opción un mínimo acuerdo.

\section{Las mujeres que parecen débiles son más atractivas?}

Antes de Programa los alumnos pensaban "que las mujeres que parecen débiles son más atractivas”, con un mínimo de acuerdo de 9,6\% y un máximo de acuerdo de 33,3\%, después del Programa modificaron con un mínimo de acuerdo de 23,6\% y un máximo de acuerdo en 4,8\%, esto es, los alumnos modificaron su creencia positivamente.

\section{Análisis. Situaciones sufridas como víctimas, agresores, observadores Como víctima}

Los estudiantes antes y después del Programa se ignoraron menos, el 33\% que mencionó que nunca los ignoraban, cambió dos meses más tarde a $57,2 \%$.

Después del Programa los alumnos fueron menos rechazados; antes del Programa el 9,5\% nunca se sentía rechazado y el 33,3\% casi nunca después del Programa el 52,4 \% nunca se sentían rechazados y el 38,1\%, casi nunca.

Después del Programa los estudiantes percibieron más participación equitativa; antes del Programa 9,5\% de los alumnos mencionaron que no habían compañeros que les impedían participar, después del Programa ese porcentaje subió a 52,3\%, por lo que podemos argumentar que los alumnos tuvieron más oportunidad de interactuar en igualdad.

El porcentaje de alumnos que no recibieron insultos durante el desarrollo del año escolar después del Programa fue de 52,4\%, a comparación del 14,3 \% al iniciar los talleres. Lo cual nos indica que los estudiantes lograron mejorar su forma de comunicarse.

También hubo una disminución en el uso de sobre nombres, antes y 
después del Programa de intervención; del 33,2\% de alumnos que nunca eran llamados por sobre nombre, se incrementó al 42,9\%. Pero seguimos teniendo un porcentaje alto.

Después del Programa los estudiantes manifestaron que les rompían menos sus cosas; el 4,8 \% que mencionó que nunca les rompían sus cosas, se modifico a $47,6 \%$.

Los estudiantes después del Programa manifestaron que les roban menos las cosas; del 14,3\% de los estudiantes que manifestaron que no sufrieron ese problema, subió al 42,9\%, por tanto el análisis de los talleres permitieron a los estudiantes analizar sus actitudes y modificarlas.

\section{Como agresor}

Los alumnos agresores, describen que después del Programa ponen menos apodos que ofenden a sus compañeros, lo que implica que los talleres fomentan formas más adecuadas de comunicarse y jugar entre ellos.

Como agresor los estudiantes también manifestaron mejoras en su comportamiento; del 9,5\% de estudiantes que mencionaron no agredir, se modificó al 42,9\%. Por lo que el análisis realizado en los talleres permitió a los estudiantes comprender que no es empático agredir a los compañeros.

Después del Programa los estudiantes agresores, modificaron positivamente sus actitudes con respecto a dejar participar equitativamente a sus compañeros; el 19\% que mencionó que nunca impidió participar, después del Programa se incrementó a 28,9 \%.

Los alumnos también describen que después del Programa, lograron modificar un poco la costumbre de hablar mal de sus compañeros, por ejemplo, del 38,1\% de estudiantes que hablaban mal de sus compañeros cambio a $4,8 \%$.

La actitud de pegarle a sus compañeros se modificó positivamente después del Programa; el porcentaje de alumnos que pegan, cambió de un $19 \%$ a un $9,5 \%$.

\section{Como observador}

Como observadores los alumnos dicen haber percibido más violencia después del Programa que antes del Programa. La respuesta se nos hace interesante porque es posible que se volvieran más observadores con respecto a qué es violencia y qué no. La categoría se modifico de $71,4 \%$ a $61,9 \%$.

Los alumnos observaron mayor participación equitativa, es decir, se permitían participar. El porcentaje de respuesta más positivo se modifico de $33,3 \%$ a $57,1 \%$.

Los estudiantes observaron que se pegaron menos entre alumnos durante el proceso de comunicación, lo que indica que encontraron formas 
más adecuadas de molestarse, llamar la atención y, resolver sus problemas. El 52,4\% por ciento de los estudiantes después del Programa observaron menos golpes entre los compañeros.

\section{Análisis. Creencias de los adolescentes hacia las conductas violentas, relaciones interpersonales, comunicación valores, manejo de sentimientos y emociones}

Los resultados nos indican que es los alumnos necesitan conocerse más; el 57,1\% piensa o siente que sus amigos y él son desconocidos. Los alumnos comentan que cuando tienen problemas casi nunca se los comunican a sus amigos; el 61,9\% dice que casi nunca lo hace y el 38,1\% dice que nunca.

El analizar en los talleres la importancia de pensar en positivo, y observar los hechos buenos, y no solo los negativos, tuvo resultados adecuados; antes del Programa sólo el 9,5\% pensaba cosas positivas durante el día, este porcentaje se modificó a 57,1 \%.

Hubo cambios positivos después del Programa con respecto a la agresividad de los estudiantes cuando les dan una respuesta inadecuada. Antes del Programa el 14,3\% eran agresivos, cambiando este porcentaje a 4,8\% después del Programa.

Después del Programa los alumnos fueron más tolerantes y participativos, en diálogos en los cuales no estaban de acuerdo. Del 9,5 \% de estudiantes que participaban en conversaciones en las que no concordaban, se modificó a $61,9 \%$.

Los estudiantes desarrollaron sus habilidades de comprensión del lenguaje no verbal, en los talleres se estuvo analizando la importancia de leer el lenguaje corporal y los gestos, para comprender mejor a las personas. Antes del Programa el 47,6 \% de los estudiantes que podían leer la expresión de la cara de sus amigos, se modificó a 66,7\%.

Durante el Programa se trabajó con los estudiantes la importancia de buscar las palabras correctas para comunicarse, se les sugirió que si tenían que decir algo pensaran sus palabras. Antes del programa el 14,3\% de los estudiantes podían expresarse claramente, después del Programa el 33,3\% mencionó poderlo hacer

Otro de los aspectos que se trabajó durante el Programa fue poder expresar el enojo sin lastimar al otro, se pusieron ejemplos y se adecuaron actividades didácticas para analizar esta situación. Los resultados fueron positivos del 23,8\% que lograban expresar sin lastimar, cambió al 42,9\% de alumnos que lo lograron hacer, después de los talleres.

El respeto es uno de los valores que más analizamos; antes del Programa el 14,3\% de los estudiantes valoraban el respeto, después del Programa el 28.6\% mencionaron hacerlo. 
Una habilidad relevante para resolver problemas es describirlos, analizar la causa; antes del Programa el 19\% de los estudiantes lo lograba hacer, después del Programa el 57\% menciona hacerlo.

El proceso de solución de problemas implican varias fases, el tener soluciones alternativas es trascendental. Antes del Programa el 19\% de los alumnos buscaban soluciones alternativas, después del Programa el 52,4 \% mencionó hacerlo.

Es importante que aprendamos de nuestros errores, por lo que fue uno de los aspectos que analizamos durante todos los talleres; antes del Programa el 23,8\% de los alumnos mencionó que aprendían de sus errores, después del Programa el 38,1\% lo logró.

Para poder comunicarnos sin violencia, uno de los aspectos relevantes es desarrollar nuestra inteligencia emocional, comprender qué sentimos y por qué sentimos; antes del Programa el 57,1\% de los estudiantes se daba cuenta de lo que sentía, después del Programa el 47,6 \% dijo darse cuenta. El porcentaje fue menor, lo que pudo haber ocurrido es que este proceso de comprender nuestros sentimientos y emociones no es sencillo, y al darse cuenta lo que implica, los estudiantes no se mostraron seguros en decir que sabían lo que sentían. Se requiere de mayor formación en inteligencia emocional.

Es importante manejar las emociones, esto es, por ejemplo, cuando están enojados para evitar generar violencia. Antes del Programa el 4,8\% de los estudiantes lograban contener su respuesta cuando estaban enojados, después del Programa mejoró al 28,6\%.

El autoconocimiento permite una comunicación más asertiva, así como la aceptación de las actitudes de reconocer que todos tenemos errores y conocer los propios. Antes del Programa el 47,6\% de los estudiantes reconocieron sus errores, después del Programa el 57,1\% lo hicieron.

4.5 Análisis cualitativo del proceso a través de los portafolios elaborados por los estudiantes que participaron en el Programa.

A través de las autoevaluaciones, tareas y actividades se logró analizar el proceso de los estudiantes durante el Programa de intervención. Se seleccionaron la respuestas más representativas y no se escribieron aquellas que eran muy parecidas. Entre las metas cumplidas que describieron podemos comentar las siguientes:

Testimonios: Cambié mi forma de ver la violencia; empecé a: pensar en los demás, ser tolerante, tener paciencia; Me conocí cómo soy; Me comunico mejor; Mis relaciones con los demás son mejores; Me divertí.

Los estudiantes expresaron que les gustaron los talleres porque: "Me gustaron las dinámicas"; "Los talleres fueron muy importantes ya que ayudaron a arreglar y cambiar muchas cosas"; "Fueron muy divertidos"; "Fue muy lindo todo, pero no creo que los niños cambien con esto, muchas 
gracias; "Aprendimos y nos divertimos al mismo tiempo"; "Utilizaron una forma interesante de aprender y nos va a ayudar cuando tenemos problemas";

Por otro lado en una de las tareas a realizar se les preguntó que mencionaran los mensajes inadecuados que habían dicho en la semana y cómo los habían corregido; se obtuvieron respuestas como: le pedí perdón, traté de utilizar otras palabras, estoy tratando de pedir permiso cuando me encuentro con alguien que me obstaculice.

En una de las lecturas que se les dejo, llamada "Asamblea de herramientas", en la cual se promueve, la tolerancia, el trabajo cooperativo, el respeto, entre muchos otros aspectos relevantes para una cultura de paz, los estudiantes nos contestaron que habían aprendido lo siguiente: hay que mirar lo positivo, todos tenemos defectos y no hay que enfocarse en ellos, no importa las diferencias, siempre vas a ser útil, a pesar de sus defectos pudimos trabajar con sus cualidades, tenemos que ser comprensivos y respetuosos, no debo ofender.

Se quisieron analizar los sentimientos que experimentan los estudiantes en su vida cotidiana, durante una semana se les puso la tarea de analizar, qué sentían y qué emociones reconocían, y los resultados fueron: tristeza, dolor, rabia, intolerancia, felicidad, celos, amor, alegría, enojo, ira, depresión. Llama la atención que si hiciéramos un análisis de cuáles son positivos y cuáles negativos, los estudiantes mencionan mucho más los segundos; lo que implica que a esta edad en donde ellos pasan por múltiples cambios, requieren un acompañamiento que les permita la comunicación, el análisis de emociones y actitudes, entre otras.

También tuvieron la oportunidad de analizar sus defectos y sus virtudes. Entre los defectos mencionaron: intolerante, impulsivo, distraído, malhumorado, desconfiado, temperamental, enojón, inquieto, amargado, tímido, insociable, vaga, odiosa, mentiroso, sisañoso, atrevido. Entre las virtudes mencionaron: inteligente, preocupado por los demás, soy feliz, cariñosa, buena amiga, tierna, fuerte, deportista, creativo, positivo, responsable, confiable, exitoso.

Se trabajaron distintas competencias estructuradas en habilidades, valores y actitudes se que desarrollaron durante los talleres; entre las habilidades están: "asertivas, empatía, respeto, comunicación”; entre los valores: "respeto, amistad, tolerancia, trabajo en grupo"; entre las actitudes se mencionan: "respeto hacia los compañeros, tolerante, positiva, responsable, apoyo a mis compañeros, colaborativa".

Uno de los puntos relevantes de analizar era qué harían los estudiantes si tienen un amigo en el salón que está siendo agredido; él es inseguro y callado y no se atreve a defenderse, ¿qué harías? ¿cómo solucionarías el problema? Respuestas: les diría que lo dejen, hablaría con 
mis compañeros, hablaría con los profesores, le ayudaría a defenderse, defenderlo y tratar de conseguirle amigos, hablar con la coordinadora y decirle el nombre de los compañeros que lo están molestando. Es interesante analizar que todos los estudiantes fungen distintos roles, como agresores o observadores en el contexto escolar cotidiano, pero todos describieron respuestas solidarias a su compañero. Se argumenta que cuando son agresivos, no lo quieren ser, sólo reaccionan; Si se les pregunta sobre un problema de violencia en concreto, su opinión y análisis es de apoyo, empatía, protección y amistad.

\section{Conclusion y recomendaciones}

La investigación realizada valora el Programa que se basa en la promoción de la cultura de paz, desde una perspectiva psicológica y de salud mental en la escuela. A partir de los resultados obtenidos se tienen más elementos para resaltar la importancia del Programa de Prevención a la Violencia como parte de los temas extracurriculares que deben insertarse en el contexto académico de forma permanente. Igualmente se enfatiza la necesidad de que se hagan evaluaciones diagnósticas para reconocer la problemática sobre la violencia de los alumnos en los salones y no solo de las sugerencias de expertos muchas veces ajenos al problema concreto del alumnado.

Los estudiantes participantes expresaron que “el Programa fue divertido, les ayudó a mejorar las relaciones con sus compañeros, a resolver problemas y a aprender muchas cosas que van a servir para el futuro“

La presente investigación muestra que la violencia escolar sólo podrá resolverse promoviendo actividades que difícilmente se den en el proceso académico de forma espontánea; en las que se produzca el trabajo colaborativo con la suficiente duración e intensidad para establecer relaciones interpersonales sanas, tolerantes y respetuosas.

Concordando con lo que dice Lumsden (2000) que para que una sociedad tenga una cultura de paz requiere modificar el sistema de creencias, mediante la promoción y adopción de normas y valores de cohesión social y cooperación.

Otro aspecto que se evidencia es que los estudiantes tienen creencias aprendidas que requieren de ser modificadas para promover una cultura de paz. Como dice Corozzo (2010) las experiencias van formando nuestras creencias.

Los estudiantes fueron cooperativos, y lograron el trabajo colaborativo durante las distintas actividades del Programa, lo cual concuerda con lo expuesto por Ortega (2004), quien destaca la cooperación como la vía más eficaz para que los estudiantes aprendan a relacionarse sanamente entre ellos. 
El análisis de los resultados defiende la pertinencia de la propuesta: 1) se consiguió el objetivo de la investigación en cuanto que el grupo estudio, después de la participación modificó su conducta violenta y de bullying. 2) las evidencias recogidas en el portafolio (Inc. 4.5) que ejemplifican y detallan el proceso; 3) En el mismo apartado aparecen opiniones de los alumnos; evaluaron el proceso formativo y la metodología empleada como útil para cambiar sus creencias y prácticas violentas.

\section{Limitaciones y dificultades para lograr alumnos que se alejen del "bullying"}

El estudio evidencia la necesidad de formación de comunidades educativas conformadas con docentes, padres de familia y el personal administrativo.

Desde la perspectiva de prevención de la salud mental, se debe intervenir en la población, de forma asertiva y eficiente, para lograr disminuir las consecuencias tanto psicológicas como físicas de un entorno violento.

El contexto educativo debe ser un entorno favorecedor del desarrollo del ser humano, pues se fundamenta en el trabajo colaborativo de expertos, que tienen las competencias más adecuadas para promover el crecimiento personal asertivo y por tanto sano.

Tradicionalmente en Panamá, los currículos de formación docentes y del personal administrativo de los colegios, no contemplan temas como solución de problemas, autoconocimiento, cultura de paz, comunicación asertiva, inteligencia emocional, manejo de sentimientos y emociones, diversidad, relaciones interpersonales, entre otros, que son los que nos permiten conocernos más, aceptar a los demás y por tanto poder ejercer una cultura de no violencia, solucionando nuestros problemas y teniendo actitudes asertivas, prudentes, y adecuadas para respetar a los demás en una sociedad.

El trabajo empírico realizado ha sido cuidadosamente analizado y creemos que el Programa de Prevención de la Violencia y Promoción de Cultura de Paz desde la perspectiva psicológica y de salud mental es una herramienta valida y útil para prevenir la violencia escolar promoviendo una cultura de paz que favorezca un ambiente sano y adecuado de desarrollo personal.

Somos conscientes de que hay muchos aspectos de la investigación sobre Programas de prevención a la violencia que siguen sin cubrirse después del estudio. Aunque nuestra investigación tuvo resultados que consideramos positivos, identificamos ciertas limitaciones.

Una de ellas es que el Programa se lleva a cabo durante dos meses del proceso escolar y por tanto podemos argumentar cambios en las creencias, 
más no cambios permanentes en las actitudes pues para ello se requeriría de más acompañamiento de los estudiantes durante el proceso académico, además de evaluaciones posteriores para medir si los cambios son permanentes.

Por otro lado sólo se pudo trabajar por situaciones de tiempo y cantidad de estudiantes con dos grupo; hubiera sido interesando poder medir varios grupos y de esta forma analizar si el Programa funcionaba igual en varios niveles educativos.

El Programa no constituye un conjunto cerrado y rígido de prescripciones para que el estudiante siga las reglas. Cada alumnos tiene su familia y su educación recibida propia y por tanto no es necesario ni conveniente un manual pero los docentes deben incluir a los alumnos en las decisiones de cómo se deben comportar y cómo evaluar sus comportamientos. Este carácter no encerrado supone un esfuerzo de concreción curricular y metodología que debe añadir a su carga de trabajo, ya de por si abultada.

En síntesis, los resultados de la evaluación diagnóstica indicaron aspectos sobresalientes en los que hay que trabajar antes, durante y después de los cursos académicos. A partir de esos resultados fue la propuesta del Programa, que en definitiva requiere ser perfeccionado continuamente a fin de enriquecerlo y que se vaya modificando de acuerdo con las necesidades de los profesores, estudiantes y la familia de manera que no se vuelva ineficiente como muchos de los programas que se ofrecen cada año.

\section{References:}

Bandura, A., (1986). Social Foundations of Thought and Action: A Social Cognitive Theory. Englewood Cliffs, N.J.: Prentice-Hall.

Bisquerra, R. (2003) Educación emocional y competencias básicas para la vida. Revista de investigación educativa. Vol 21, 1

Bullying Prevention Program (2014) https://www.bullyfree.com

CEASPA (2013), Violencia en las escuelas de Panamá. www.unicef.org/panama/spanish/media_25480.htm

Corozzo, J.,(2010) El bullying en la escuela. Psicología.12,329-346.

Comunicado de Madrid, (1999).www.uv.es/ pla/CCOO/lru/pdi78-2.html

Cruz Roja (2003) Estrategias Educativas para la prevención a la violencia: mediación y dialogo. España: juventud, Cruz Roja.

Del Rey ,R., Ortega, R., (2001) programas para la prevención de violencia en España. La respuesta de las comunidades autónomas. Revista interamericana de Formación de profesorado.41.

Díaz-Aguado, M.,(2003) La violencia en la Escuela. Sanmartín. Barcelona: Ariel. 
Díaz-Aguado, M., (2004) Las causas de la violencia desde una perspectiva Ecológica. Manuscrito interno, Universidad Complutense de Madrid.

Díaz-Aguado, M., Martínez, R., Martín, G., (2002): Génesis y desarrollo de los comportamientos de los jóvenes con problemas de conducta en centros de menores. Madrid: Ministerio de Trabajo y Asuntos Sociales.

Díaz-Aguado, M., (2004): Investigación: Prevención de la violencia y lucha contra la exclusión desde la adolescencia. Madrid: Instituto de la Juventud.

Díaz -Aguado, M., Martínez, R., Martin, G., (2004) Prevención de la violencia y lucha contra la exclusión desde la adolescencia, volumen uno: la violencia entre iguales en las escuela en el ocio. Estudios comparativos e instrumentos de evaluación. Madrid: Instituto de la juventud.

Eljach, S., (2011) Violencia escolar en América Latina y el Caribe. Superfice y fondo. Panamá: UNICEF.

Green, J., (1971) The activities of teaching. Nueva York: McGraw-Hill.

Guerrero, H., (2015) El portafolio: Una herramienta facilitadora del cambio en la educación superior desde la practica didáctica. Revista Zona Próxima, 22, enero-junio, pp 143-155.

Gómez, A., Gala,F.J.,Lupiani, M., Bernalte,B., Miret,M.,Lupiani,S., Barreto,M. (2007) Bullying y otras formas de violencia adolescentes. Cuadernos de Medicina Forense. N.48-49,abr-jul. ISSN 1135-7606.

Hart , L., (1989) Describing the effective domain: Saying what we mean. En McLeord, D., Adames, V., Affect and Mathematical problema solvin. Nueva York: Spinger-Verlarg.

Hernández, R., (2010) Metodología de la Investigación. México: McGrahill. INJUVE, (2010) Prevención a la violencia y lucha contra la exclusión desde la adolescencia. España: Instituto de la Juventud.

Kerman, B., (2016). Un estudio descriptivo de las creencias docentes acerca del fenómeno Bullying. European Scientific Journal./SPECIAL/edition. 2829.

Kerman, B. (2015). Estudio de eficacia de un programa de mejora en el manejo del hostigamiento entre pares (Bullying) en el ámbito aúlico en docentes de nivel primario de la Ciudad de Buenos Aires. Estudio de las creencias de los docentes sobre los factores y acciones preventivas. European Scientific Journal./SPECIAL/edition. 2, 176.

Lumsden, M., (2000) "Engendering Peace: Creative Arts Approaches to Transforming Domestic and Communal Violence", en I. Breines, R. Connell y I. Eide. Male Roles, Masculinities and Violence. A Culture of Peace Perspective. París, UNESCO, 2000, 257-270.

Morollon, M., (2001) Una panorámica sobre la convivencia en los centros. Cuadernos de Pedagogía, 304,42-54.

Organización Mundial de la Salud (2002).Informe mundial sobre la violencia y la salud. Washington, D.C.: OMS. 
Ortega y Gasset J., (1989) Ideas y Creencias. Revista de Occidente. México, Volumen V.

Ortega, R. (2000). A Global, Ecological and Cultural Model for Dealing with Problems of Violence in European Compulsory Schools. Paper presentado en 6th Meeting of TMR Programme Nature and Prevention of Bullying and Social Exclusion. Cruz Quebrada-Dafundo. Lisboa.

Ortega, R.,(2003) Convivencia Escolar: qué es y cómo abordarla, programa educativo de prevención de maltrato entre compañeros. España: Consejería de Educación y Ciencia. Junta de Andalucía.

Ortega R., (2004) Educar la convivencia para prevenir la violencia. Madrid: Antonio Machado libros.

OMS(1996) Carta de Otawa: Conferencia Internacional sobre la promoción de la Salud. Canadá: OMS.

OMS(1997) Declaración de Yakarta: Conferencia Internacional sobre la promoción de la Salud. Indonesia: OMS.

OMS (1998) Promoción de la salud Mental - Glosario. España: Ministerio de Sanidad y Consumo.

Pajares, F., (1992) Mathematics Teachers Professional Knowledge. En Ponte, J.,Matos, J., Proceedings of the eighteenth international conferences for the psychology of mathematics education. Portugal: International Group for the psichology of mathematics education.

Pinhjeiro, P. (2007). Acabar con la violencia contra los niños, niñas y adolescentes (en línea): www.unicef.org/lac/capitulo0(2).pdf

Murillo, F. ( 2011) Metodología de investigación en Educación Especial Madrid: Universidad Autónoma de Madrid.

Torrego, J., (2000) La ayuda entre iguales para mejorar la convivencia escolar. España: Narcea.

Tuvilla, R.,(2004) Guía para elaborar un Proyecto integral de Escuela: Espacio de Paz ". Sevilla: Consejería de educación de la junta de Andalucía.

UNICEF,2011. Informe de Violencia escolar en América Latina y EL Caribe, www.unicef.org/lac/violencia_escolar_OK.pdf

Young (1969) Psicología social de la personalidad. Buenos Aires: Paidós 